\title{
Galectins: New agonists of platelet activation
}

Mirta Schattner ${ }^{1}$ and Gabriel A. Rabinovich ${ }^{2,3}$

1- Laboratory of Experimental Thrombosis, Institute of Experimental Medicine (IMEX/CONICET), National Academy of Medicine, C1425, Buenos Aires, Argentina

2- Laboratory of Immunopathology, Institute of Biology and Experimental Medicine (IBYME/CONICET), C1428, Buenos Aires, Argentina

3- Laboratory of Structural and Functional Glycomics, School of Exact and Natural Sciences, University of Buenos Aires, C1428, Buenos Aires Argentina.

Corresponding Author: Mirta Schattner. IMEX. Pacheco de Melo 3081, Buenos Aires 1425. Argentina. FAX: 5411-48050712, Phone:5411-4807 ext. 301. 


\section{Summary}

Platelet activation at sites of vascular injury leads to the formation of a hemostatic plug and is crucial for hemostasis. However, uncontrolled platelet activation may lead to the formation of occlusive thrombi. Several soluble or matricellular proteins can activate platelets. In this article we review recent advances on the role of galectins in platelet physiology. Either in soluble or immobilized form, these endogenous glycan-binding proteins trigger platelet activation through modulation of discrete signaling pathways. We discuss the role of platelet-galectin interactions not only in hemostasis, but also in chronic inflammation, atherosclerosis and cancer.

Keywords: platelets, galectins, hemostasis, atherosclerosis, cancer, inflammation. 


\section{Platelets}

Platelets are anucleated cell fragments released from megakaryocytes. The primary role of platelets is to initiate and maintain haemostasis. However, their function is not limited to thrombosis, and extends into physiological processes such as tissue repair, wound remodeling and antimicrobial host defense, or pathologic processes such as atherosclerosis, chronic inflammation or cancer (Wagner 2005; Rivera, Lozano et al. 2009; Leslie 2010). In all these processes, platelets act as sentinels capable of responding rapidly to chemical changes in their environment. Under physiological conditions, platelets circulate in the blood in a quiescent state and their activation is inhibited by both, nitric oxide and prostacyclin released from endothelial cells. However, after vascular injury, platelets become activated and provide rapid protection against bleeding and catalyze the slower formation of stable blood clots via the coagulation cascade. In the initiation phase of primary hemostasis, platelets roll, adhere, and spread on the collagen matrix to form an activated platelet monolayer. During the rolling phase, adhesion is mediated mainly by the interaction between the glycoprotein (GP) lb/V/IX receptor complex on the platelet surface with von Willebrand Factor (VWF), and between the GPVI and integrin lalla proteins with collagen present in the exposed subendothelium. These interactions allow the arrest and activation of adherent platelets. Platelet adhesion triggers the release of soluble mediators such as adenosine 5-diphosphate (ADP), thromboxane $A_{2}\left(T_{X} A_{2}\right)$ and serotonin, which in combination with the locally generated thrombin, allow the formation of platelet-platelet aggregates and the 
recruitment of additional circulating platelets which extend and stabilize the hemostatic plug. Specific interactions of these agonists with their G-protein coupled receptors generate inside-out signaling leading to the conformational activation of integrins, in particular $\alpha \mathrm{Ilb} \beta 3$, increasing their ligand affinity (Angiolillo, Ueno et al. 2010). Binding of $\alpha \mathrm{Ilb} \beta 3$ to its ligands, mainly fibrinogen, supports processes such as clot retraction, platelet aggregation and secretion of the granule proteins (Ma, Qin et al. 2007; Rivera, Lozano et al. 2009). Platelets contain four types of storage compartments - $\alpha$-granules, dense granules, peroxisomes and lysosomes - whose contents are released into the circulation. When stimulated by thrombin, the platelet releasate contains $>300$ proteins including prothrombotic substances, growth factors, chemokines and proteases. Although the release of cargo during exocytosis delivers many proteins into the circulation, the process also alters the composition of the platelet membrane, resulting in surface expression of $\mathrm{P}$-selectin and an increase in the number of integrin $\alpha$ llbß33 molecules (Nurden, Nurden et al. 2008). The exposure of P-selectin is especially important for platelet-leukocyte and platelet-endothelial interactions, as this molecule mediates the initial interactions of leukocytes and endothelium with activated platelets (Wagner 2005).

The ability of platelets to store and release such a variety of bioactive mediators allows them to play an important role in modulating the function of other cells and explains why platelets are also critically involved in other biological processes beyond hemostasis.

\section{Galectins}


Galectins are evolutionarily-conserved lectins defined by at least one carbohydrate recognition domain (CRD) with affinity for $\beta$-galactosides and conserved sequence motifs. These lectins are found in almost all organisms and to date 15 galectins have been found in mammals; although only 12 galectin genes are found in humans. On the basis of their molecular structure, galectins are classified into three main types: 'proto-type' galectins, comprising a single polypeptide chain that is able to dimerize (galectin-1, -2, -5, -7, -10, -11, -13, -14, and -15); "tandem repeat-type" galectins, composed of a single polypeptide chain presenting two CRDs connected by a linker peptide (galectin-4, -6, -8, -9, and -12); and the "chimera-type" galectin-3, which consists of one C-terminal CRD linked to an Nterminal peptide. A more recent classification based on gene sequence comparisons and intron-exon position analysis has classified galectins' CRDs into two distinct subgroups (F3 and F4). Peculiarly, bi-CRD galectins usually display one domain of each type, F3 and F4 (Di Lella, Sundblad et al. 2011). Although most galectins bind $\mathrm{N}$-acetyllactosamine [Galß1-4-NAcGlc] units, important differences in glycan binding preferences among different members of the family have been reported (Rabinovich and Toscano 2009). Galectins function, either intracellularly or extracellularly, as key biological mediators capable of monitoring changes occurring on the cell surface during fundamental biological processes such as cellular communication, inflammation, wound healing, development, and differentiation. They also play unique roles in intracellular signaling pathways, regulation of RNA splicing, cell cycle control, and control of apoptotic signaling, endocytosis machinery, and trafficking (Di Lella, Sundblad et al. 2011). Galectins 
can influence the capacity of innate immune cells (e.g., neutrophils, dendritic cells, monocytes/macrophages, eosinophils, and mast cells) to respond to chemotactic gradients, migrate across endothelial cell surfaces, synthesize and release both pro- and anti-inflammatory mediators, and recognize, engulf, and kill microbes and damaged cells. In this regard, similar to alarmins some members of the galectin family contribute to trigger innate immune responses, while others influence the resolution of acute inflammation. Besides, galectins can bind glycans on the surface of pathogenic microbes coupling recognition processes to innate immunity (Davicino, Elicabe et al. 2011).

In spite of lack of a typical leader peptide required for secretion, galectins are released onto the extracellular milieu through an unusual, mostly unresolved mechanisms, where they play key roles as soluble mediators of various cell functions. In fact, galectins have been proposed to exert biological activities by forming supramolecular structures, termed "lattices" with cell surface $\mathrm{N}$-and $\mathrm{O}$ glycans (Rabinovich, Toscano et al. 2007)

Most galectins are expressed in a wide variety of tissues. While some galectins (e.g., galectin-1 and -3) are widely expressed among different tissues of various species, other family members have a more restricted tissue localization and compartmentalization (e.g., galectin-7 is preferentially found in the skin, galectin-12 abundantly expressed in adipose tissue, galectin-5 restricted to rat reticulocytes, and galectin-10 strongly represented in human eosinophils, where they play essential roles in the control of cell fate (Di Lella, Sundblad et al. 2011). Most galectins are overexpressed in several types of tumors and their expression correlates with tumor progression, aggressiveness and metastatic phenotype (Liu 
and Rabinovich 2005). For example, galectin-1 overexpression contributes to tumor immune escape, modulates tumor cell migration and adhesion and influences tumor angiogenesis (Thijssen, Postel et al. 2006). High expression of galectin-8 is associated with malignancy in some type of tumors such as colon and lung and, preliminary studies showed that inhibition of galectin-8 promotes tumor regression (Bidon-Wagner and Le Pennec 2004).

\section{Platelet Activation Responses Induced by Galectin- 1 and -8}

Hemostasis and pathological thrombus formation are dynamic processes that require a co-ordinated series of events involving platelet membrane receptors, bidirectional intracellular signals, and release of platelet proteins and inflammatory substances. Platelet activation can be triggered by a broad spectrum of vascular adhesive proteins such as VWF, collagen, fibronectin and soluble agonists including ADP, TXA2, thrombin and serotonin. (Rivera, Lozano et al. 2009). It has been recently shown that two structurally different members of the galectin family of proteins, galectin-1 and 8 are potent platelet agonists (Pacienza, Pozner et al. 2008; Romaniuk, Tribulatti et al. 2010; Romaniuk, Croci et al. 2012). Through the interaction with the carbohydrate backbone of the major platelet receptors involved in hemostasis, e.g.: GPIbVIX complex and integrin $\alpha$ llb $\beta 3$, both galectins are capable to induce all the myriad of platelet responses including adhesion and spreading, aggregation, release of their granule content and the expression of $\mathrm{P}$ - 
selectin on the platelet membrane (Pacienza, Pozner et al. 2008; Romaniuk, Tribulatti et al. 2010; Romaniuk, Croci et al. 2012).

\section{Platelet receptors for Galectin-1 and Galectin 8}

Most of the extracellular functions mediated by galectins involve the interaction of these proteins with different cell surface glycoconjugates containing repeating units of $\mathrm{N}$-acetyllactosamine [Galß1,4GlcNAc] as well as extracellular matrix proteins . Among the different receptors, integrins are known to be involved in galectin-

mediated biological responses (Rabinovich, Toscano et al. 2007). Platelet activation mediated by galectin- 1 or galectin- 8 is prevented by lactose but not by sucrose indicating the interaction of these lectins with specific carbohydrate ligands on the platelet surface. MALDI/TOF mass spectrometry analyses determined that both galectins binds the subunit $\alpha$ llb from the $\alpha$ llb $\beta 3$ integrin (Pacienza, Pozner et al. 2008; Romaniuk, Tribulatti et al. 2010). Additionally, while galectin-8 also binds GPIb and V from GPIb-IX-V complex (Romaniuk, Tribulatti et al. 2010), galectin-1 interacts with $\alpha 6 \beta 1$. However, the use of platelets derived from patients who are deficient in $\alpha \mathrm{llb} \beta 3$ or in GPIb, revealed that only GPIb and integrin $\alpha$ llb $\beta 3$ are essential for galectin-8- and 1 dependent signal transduction respectively, and therefore represent functional counter-receptors (Romaniuk, Tribulatti et al. 2010; Romaniuk, Croci et al. 2012).

Similar to other integrins, the function of platelet $\alpha$ llb $\beta 3$ is regulated by inside-out or outside-in signaling. Inside-out signaling occurs in response to binding of one or 
more platelet agonists and leads to the conversion of allb $\beta 3$ from a lowaffinity/avidity receptor to a high-affinity/avidity receptor, thereby allowing engagement of soluble multivalent adhesive ligands, such as fibrinogen and vWF. Ligand binding to the integrin triggers another cascade of signals known as outside-in, which leads to irreversible adhesion and aggregation (Ma, Qin et al. 2007). In this context, galectin-1 acts as a peculiar agonist as it is capable of triggering platelet activation through outside-in signaling via direct binding to allb $\beta 3$ integrin. This intrinsic property of galectin-1 may be associated to a general feature of galectins, which has been proposed to form ordered arrays of supramolecular structures composed of multivalent glycans and lectins (often termed "lattices") on the cell surface (Yang, Rabinovich et al. 2008). Thus, it is conceivable that galectin-1 binding to allb $\beta 3$ integrin forms lattices that induce integrin clustering that leads to platelet signaling and activation.

\section{Signaling Pathways involved in galectin-mediated platelet activation}

Although the model of how GPIb-GPIX-GPV signals in human platelets is currently incomplete, the activation of Src, PI3K, PLCY 2 and ERK appears to be involved in GPlb activation mediated by vWF binding, its major ligand (Ozaki, Asazuma et al. 2005). In accordance with the notion that GPIb is an essential receptor for transducing galectin-8 signaling, platelet stimulation by galectin-8 results in phosphorylation of PLCY2, ERK1/2 and Akt. The phosphorylation of PLCY2 and Akt appear to be relevant for platelet activation induced by galectin-8 since platelet aggregation is impaired in the presence of specific inhibitors of PLC 
and PI3K but not of MAPKK (Romaniuk, Tribulatti et al. 2010).

Downstream signals involved in galectin-1 binding to platelets include the most common signals elicited by soluble or adhesive agonists that induce activation of $\alpha_{\| b} \beta_{3}$ integrin, such as $\mathrm{Ca}^{2+}$ mobilization and phosphorylation of PLCY, Syk, mitogen-activated protein kinases (MAPKs), Akt, and $\beta_{3}$ integrin. Tyrosine phosphorylation of the integrin tyrosine cytoplasmic motif of the $\beta_{3}$ subunit is one of the most proximal and specific signaling events occurring after ligand binding to $\alpha_{\| b} \beta_{3}$ integrin and it is evident in vitro only under stirring conditions that allow integrin clustering or on platelet adhesion to immobilized fibrinogen (Ma, Qin et al. 2007). Galectin-1-induced phosphorylation of $\beta_{3}$ integrin occurs rapidly in addition of galectin-1 to platelet suspensions. Moreover, rapid tyrosine phosphorylation of Syk, PLCY2, and Akt (signaling events involved in platelet outside-in signaling) was also observed after platelet exposure to soluble galectin-1 (Romaniuk, Croci et al. 2012). Interestingly, phosphorylation of these kinases has been described on platelet stimulation with concanavalin $A$, a plant lectin with different glycan specificity but similar conformation and topology that triggers platelet activation by inducing clustering of $\alpha_{\| b} \beta_{3}$ integrin molecules (Torti, Festetics et al. 1999).

\section{Galectin-1 and galectin-8 trigger platelet aggregation}

The capacity of platelets to form a thrombus depends on their ability to aggregate. At the molecular level, platelet aggregation is mediated by a specific receptor on the platelet surface: the allbß3 integrin (Rivera, Lozano et al. 2009). Like most traditional platelet agonists, both soluble galectin- 1 and galectin- 8 promote the 
transition of this integrin from a low-affinity state (resting state) to a high-affinity state (active state), which results in the unmasking of neoepitopes in the allbß3 complex, and allows fibrinogen binding which acts as a bridging molecule between platelets to form aggregates. This conformational change of allb $\beta 3$ integrin triggered by galectins is accompanied by a raise in intracellular calcium levels as well as morphological changes of platelets involving the rearrangement of the cytoskeleton including extension of filopodia and lamellipodia (spreading) and Factin polymerization (Pacienza, Pozner et al. 2008; Romaniuk, Tribulatti et al. 2010).

Both, galectin-1 and galectin-8 induce aggregation of platelets suspended either in plasma or buffer, which indicates a relevant role of these lectins in physiological media. Although cell agglutination was one of the first biological activities described for these lectins, this effect on platelets was only observed at high galectin-1 and galectin-8 concentrations. Platelet aggregation at lower concentrations is absent in fixed platelets, in the presence of calcium chelating agents, or eptifibatide (an allbß3 antagonist), implying that platelet responses mediated by galectins could be related either to cell activation or to a clustering effect of platelet surface receptors depending on their concentration (Pacienza, Pozner et al. 2008; Romaniuk, Tribulatti et al. 2010). Although both galectins were capable of promoting aggregation, galectin- 8 was found to be ten times more potent than galectin-1 in this stimulatory effect. Although the dimeric structure of galectin-8 would anticipate a more robust effect of this galectin compared to galectin-1, the observation that only the $\mathrm{N}$-terminal domain was also able to trigger platelet activation indicates that lectin bivalency is not essential to promote 
activating effects on platelets. The differences in the concentration required to achieve a similar effect, could therefore reflect different downstream molecular signals triggered by each galectins (Romaniuk, Tribulatti et al. 2010; Romaniuk, Negrotto et al. 2011).

The initial formation of platelet thrombus is rapidly reinforced by the generation and release of platelet TXA2 and ADP (the main metabolite of arachidonic acid by the cyclooxigenase pathway, and a component of platelet dense granules respectively) which acting in a paracrine and autocrine manner promote further platelet activation (Rivera, Lozano et al. 2009). Both molecules are generated on platelet stimulation by galectin-1 and -8 (Pacienza, Pozner et al. 2008; Romaniuk, Tribulatti et al. 2010). Moreover, although the aggregation response triggered by low galectin- 8 concentrations was inhibited in the presence of aspirin (cyclooxigenase inhibitor) and/or an ADP-scavenger, a full response was obtained at higher concentrations indicating that galectin-8 is a strong agonist that activate platelets independently from $\mathrm{TXA}_{2}$ formation or ADP release (Pacienza, Pozner et al. 2008; Romaniuk, Tribulatti et al. 2010).

\section{Proinflammatory and procoagulant platelet-mediated responses induced by galectins}

The role of platelets in inflammation and vascular repair is mainly associated to the release of their alpha granule content, which includes among others, cell adhesion molecules that favor platelet/endothelial and leukocyte interaction, prothrombotic 
substances, and angiogenesis regulatory growth factors (May, Seizer et al. 2008; Etulain J 2012).

Galectin-1 and -8 are strong inducers of P-selectin expression (Pacienza, Pozner et al. 2008; Romaniuk, Tribulatti et al. 2010). The major role of P-selectin on the platelet surface is the interaction with PSGL-1, its major counter-receptor on leukocytes, to form platelet-leukocyte aggregates (Wagner 2005). In fact, activation of platelets by galectin-1 in the presence of polymorphonuclear leukocytes results in a significant formation of heterotypic cell aggregates (Pacienza, Pozner et al. 2008). This interaction promotes activation of both cell types, which is a crucial condition for triggering inflammation, vascular remodeling and thrombosis. The expression of P-selectin on the platelet surface, the formation of leukocyte-platelets aggregates and leukocyte activation, are relevant events in acute syndromes including coronary diseases as well as in deep vein thrombosis (DVT) (Dotsenko, Chaturvedi et al. 2007; Etulain J 2012), Given that galectins promote all these responses, binding of these lectins to platelets might represent potentially novel mechanism involved in both, arterial and venous thrombosis.

In a cancer setting, the formation of heterotypic aggregates between circulating tumor cells overexpressing galectin- 1 or -8 and platelets would result in P-selectin expression, which may contribute to the metastatic properties of these galectins by promoting stable adhesion to the endothelium and/or transmigration of tumor cells outside of the vasculature.

vWF is a large multimeric glycoprotein that allows platelet-endothelium, platelet-subendothelium and platelet-platelet interaction and is therefore important for platelet adhesion and thrombus formation. Similarly to P-selectin, vWF is stored 
in platelet $\alpha$-granules and in Weibel-Palade bodies of endothelial cells from which is released during injury or inflammation. VWF is a biomarker for endothelial dysfunction and cardiovascular risk and high levels of vWF are found in both chronic and acute inflammation (Luo, Ni et al. 2012). The release of vWF occurs after activation of platelets by galectin-1 (unpublished data) and -8 (Romaniuk, Tribulatti et al. 2010). Thus, P-selectin expression and vWF release mediated by galectins might play an important role in the pathogenesis of thrombus formation and the modulation of inflammatory responses.

While galectin-8 has been linked to proinflammatory processes (Cattaneo, Tribulatti et al. 2011), both, anti and proinflammatory activities have been described for galectin-1 (Liu and Rabinovich 2010). Whether anti-inflammatory and pro-inflammatory effects occur at different concentrations of galectin-1 still remains to be established. The fact that galectin-1 promotes platelet activation support to the notion that under certain circumstances, galectin-1 could also act as a proinflammatory factor.

Vesiculation of the platelet membrane and formation of platelet microparticles (PMPs) is a characteristic effect of certain agonists such as collagen, thrombin or C5b-9 complement fragment. PMPs are released not only during platelet activation in vitro but are also detected in vivo (Morel, Toti et al. 2006). They are thought to provide catalytic surface for several enzyme complexes of the coagulation system and to underlie the pro-coagulant responses elicited by platelet activation. Moreover, PMPs may themselves evoke cellular responses in the immediate microenvironment including platelet adhesion to the site of 
endothelial injury and angiogenesis. The ability of galectin-1 to induce phosphatidylserine-expressing PMPs (Pacienza, Pozner et al. 2008) suggests that this lectin not only promotes platelet activation, but could also indirectly activates the coagulation cascade.

\section{Galectins and platelet adhesion}

Platelet adhesion and spreading, together with cytoskeletal rearrangements, are early functional responses that occur upon vascular injury as a consequence of outside-in signaling on ligand binding to $\alpha_{\| b} \beta_{3}$ and integrin oligomerization (Rivera, Lozano et al. 2009). Platelet adhesion to the extracellular matrix at sites of vascular injury represents a key step for limiting bleeding but, if uncontrolled, can lead to occlusion. Immobilized galectin-1 and -8 are efficient substrates for platelet adhesion, filopodia, and lamellipodia formation in the absence of additional platelet agonists (Pacienza, Pozner et al. 2008; Romaniuk, Croci et al. 2012). The demonstration that immobilized galectins are as efficient as their soluble form in triggering platelet activation together with the observation that their expression is increased in activated endothelium or tumor cells (Thijssen, Hulsmans et al. 2008), suggest that the interaction of platelets with immobilized galectin-1 or -8 could be relevant in different clinical settings, including not only vascular but also inflammatory disorders such as atherosclerosis and cancer. Several studies demonstrated that galectins including galectin-1 and -8 participate in the initiation, progression and rupture of an atheroma plaque (Al-Ansari, Zeebregts et al. 2009). Galectins expressed in the inner of atheroma plaque, may not only influence 
platelet activation but may also serve as key components of the extremely thrombogenic core exposed after plaque rupture, the main trigger for acute thrombus formation and the cause of unstable angina, myocardial infarction, transient ischemic attack and stroke (May, Seizer et al. 2008).

In cancer, platelets are known to contribute to tumor progression and metastasis. One of the mechanisms underlying this effect is the formation of mixed-cell aggregates between tumor cells expressing mucins and platelets expressing P-selectin; these complexes prevent tumor cell attack by the immune system (Leslie 2010). In the same line of evidence, the interaction between tumor cells expressing high levels of galectins and platelets could represent another molecular mechanism through which both, platelets and galectins promote the metastatic cascade. Interestingly and in contrast to mucin-P-selectin interactions, the interaction of tumor cells with platelets via galectins would not require the presence of activated platelets. Furthermore, the overexpression of galectins in tumor cells could also represent a trigger for platelet activation allowing the release of $\alpha$-granules content including growth factors, which can promote tumor progression and angiogenesis.

\section{Platelets express galectin-1 and galectin-8}

In addition to vascular cells, human platelets express substantial levels of galectin1 and -8 (Pacienza, Pozner et al. 2008; Romaniuk, Tribulatti et al. 2010). Although both galectins are secreted proteins, they are mainly found in the cytosol of 
different cell types. Human platelets express the two splice variants of galectin-8. Moreover, whereas galectin-8, like P-selectin, is absent on the surface of resting platelets, it is exposed on the membrane of thrombin-stimulated platelets (Romaniuk, Tribulatti et al. 2010). This finding suggests that in the vascular system, platelets are another source of galectin- 8 that would be accessible upon platelet activation to eventually promote thrombus growth. Furthermore, it has been recently shown that galectin- 8 regulates platelet function by mediating the uptake of platelet proteins (i.e. FV) by megakaryocytes (Zappelli, van der Zwaan et al. 2012). In contrast, galectin-1 is not expressed on the platelet surface upon activation (unpublished observations). The differences between both galectins are still not clear. Interestingly, addition of lactose and thiodigalactoside partially prevents aggregation induced by classical agonists suggesting that platelet-derived galectins and their glycans might contribute to platelet activation.

Thrombosis and disseminated intravascular coagulation are common complications in cancer patients. A hypercoagulable or pro-thrombotic state of malignancy occurs due to the ability of tumor cells to activate platelets and the coagulation system. Pro-thrombotic factors in cancer include the ability of tumor cells to produce and secrete pro-coagulant/fibrinolitic substances and proinflammatory cytokines and the physical interaction between tumor cells and platelets. However, the mechanisms allowing the occurrence of prothrombotic states in cancer patients are not completely understood.

The observed increased of galectin-1 and -8 in tumoral endothelial cells as well as in other malignant cells could represent a pathogenic mechanism involved in thrombosis and disseminated intravascular coagulation complications. Given the 
pivotal role of galectin- 1 and -8 in tumor progression, it could be conceivable that galectin-induced platelet activation might contribute to the pathogenesis of thrombosis in cancer patients

\section{Galectin-1 plays a role in normal primary hemostasis}

The relevance of galectin-1 in hemostasis, has been recently studied in galectin-1 null mutant mice. Animals deficient in galectin-1 had prolonged bleeding time, an effect which was not associated with a decrease in platelet number but rather to a platelet dysfunction. In fact, galectin-1-deficient platelets showed a restricted adhesion to immobilized fibrinogen and a delayed clot retraction, two platelet activation responses dependent on outside-in signaling of platelet integrin allbß3 (Romaniuk, Croci et al. 2012). Thus, an impairment of this activation pathway appears to be a major cause of the altered bleeding time in mice lacking galectin- 1 . Interestingly, many of the hemostatic alterations present in mice with galectin-1 ablation recapitulate the phenotypes observed in mice devoid of the tetraspanin superfamily member TSSC6 or in mice in which the integrin cytoplasmic tyrosine motif has been mutated to phenylalanine, which all exhibited impaired outside-in signaling (Romaniuk, Croci et al. 2012). The concept that galectin-1 has a role in hemostasis was also recently described by Saint-Lu $\mathbf{N}$ et al. who showed that galectin-1/galectin-3 double-deficient mice are characterized by a more rapid formation of initial thrombi following ferric chloride-induced injury, but occlusive thrombi less stable compared with those of wild-type mice(SaintLu, Oortwijn et al. 2012). 


\section{Conclusions}

Over the last decade we have witnessed significant advances regarding the biology of galectins and their role in cellular homeostasis: Particularly considerable progress has been made on the contribution of galectins to innate and adaptive immune responses. The information currently available indicates that galectins are expressed and secreted by several cell types in normal and pathologic conditions. The emerging evidence showing that galectins are also capable of triggering platelet activation opens a completely new field of research where so far there are more questions than answers. From a more general perspective, this field will also uncover the role of glycans and glycosyltransferases in platelet function and hemostasis. The study of galectin-glycan interactions in platelet activation will provide further insight into the mechanisms linking inflammatory mediators to thrombus formation and could expand our view of the role of platelets much beyond homeostasis to their pathophysiologic role during inflammation and cancer.

\section{Acknowledgements}

Work in M.S's. and G.A.R's laboratories is supported by grants from the National Agency for Promotion of Science and Technology (Argentina) and National Council for Scientific and Technical Investigation (CONICET, Argentina). Work in G.A.R's 
lab is supported by Sales Foundation for Cancer (Argentina), University of Buenos Aires (Argentina), and National Multiple Sclerosis Society (USA).

\section{References}

Al-Ansari, S., C. J. Zeebregts, et al. (2009). "Galectins in atherosclerotic disease." Trends Cardiovasc Med 19(5): 164-169.

Angiolillo, D. J., M. Ueno, et al. (2010). "Basic principles of platelet biology and clinical implications." Circ J 74(4): 597-607.

Bidon-Wagner, N. and J. P. Le Pennec (2004). "Human galectin-8 isoforms and cancer." Glycoconj J 19(7-9): 557-563.

Cattaneo, V., M. V. Tribulatti, et al. (2011). "Galectin-8 tandem-repeat structure is essential for Tcell proliferation but not for co-stimulation." Biochem J 434(1): 153-160.

Davicino, R. C., R. J. Elicabe, et al. (2011). "Coupling pathogen recognition to innate immunity through glycan-dependent mechanisms." Int Immunopharmacol 11(10): 1457-1463.

Di Lella, S., V. Sundblad, et al. (2011). "When galectins recognize glycans: from biochemistry to physiology and back again." Biochemistry 50(37): 7842-7857.

Dotsenko, O., N. Chaturvedi, et al. (2007). "Platelet and leukocyte activation, atherosclerosis and inflammation in European and South Asian men." J Thromb Haemost 5(10): 2036-2042.

Etulain J, S. M. (2012). "Current viewpoints on platelet contribution to inflammation." World Journal of Hematology 1(4): 14-21.

Leslie, M. (2010). "Cell biology. Beyond clotting: the powers of platelets." Science 328(5978): 562564.

Liu, F. T. and G. A. Rabinovich (2005). "Galectins as modulators of tumour progression." Nat. Rev. Cancer 5(1): 29-41.

Liu, F. T. and G. A. Rabinovich (2010). "Galectins: regulators of acute and chronic inflammation." Ann N Y Acad Sci 1183: 158-182.

Luo, G. P., B. Ni, et al. (2012). "von Willebrand factor: more than a regulator of hemostasis and thrombosis." Acta Haematol 128(3): 158-169.

Ma, Y. Q., J. Qin, et al. (2007). "Platelet integrin alpha(Ilb)beta(3): activation mechanisms." J Thromb Haemost 5(7): 1345-1352.

May, A. E., P. Seizer, et al. (2008). "Platelets: inflammatory firebugs of vascular walls." Arterioscler Thromb Vasc Biol 28(3): s5-10.

Morel, O., F. Toti, et al. (2006). "Procoagulant microparticles: disrupting the vascular homeostasis equation?" Arterioscler Thromb Vasc Biol 26(12): 2594-2604.

Nurden, A. T., P. Nurden, et al. (2008). "Platelets and wound healing." Front Biosci 13: 3532-3548.

Ozaki, Y., N. Asazuma, et al. (2005). "Platelet GPIb-IX-V-dependent signaling." J Thromb Haemost 3(8): 1745-1751.

Pacienza, N., R. G. Pozner, et al. (2008). "The immunoregulatory glycan-binding protein galectin-1 triggers human platelet activation." Faseb J 22(4): 1113-1123.

Rabinovich, G. A. and M. A. Toscano (2009). "Turning 'sweet' on immunity: galectin-glycan interactions in immune tolerance and inflammation." Nat Rev Immunol 9(5): 338-352. 
Rabinovich, G. A., M. A. Toscano, et al. (2007). "Functions of cell surface galectin-glycoprotein lattices." Curr Opin Struct Biol 17(5): 513-520.

Rivera, J., M. L. Lozano, et al. (2009). "Platelet receptors and signaling in the dynamics of thrombus formation." Haematologica 94(5): 700-711.

Romaniuk, M. A., D. O. Croci, et al. (2012). "Binding of galectin-1 to alphallbbeta(3) integrin triggers "outside-in" signals, stimulates platelet activation, and controls primary hemostasis." Faseb J 26(7): 2788-2798.

Romaniuk, M. A., S. Negrotto, et al. (2011). "Identification of galectins as novel regulators of platelet signaling and function." IUBMB Life 63(7): 521-527.

Romaniuk, M. A., M. V. Tribulatti, et al. (2010). "Human platelets express and are activated by galectin-8." Biochem J 432(3): 535-547.

Saint-Lu, N., B. D. Oortwijn, et al. (2012). "Identification of galectin-1 and galectin-3 as novel partners for von Willebrand factor." Arterioscler Thromb Vasc Biol 32(4): 894-901.

Thijssen, V. L., S. Hulsmans, et al. (2008). "The galectin profile of the endothelium: altered expression and localization in activated and tumor endothelial cells." Am J Pathol 172(2): 545-553.

Thijssen, V. L., R. Postel, et al. (2006). "Galectin-1 is essential in tumor angiogenesis and is a target for antiangiogenesis therapy." Proc Natl Acad Sci U S A 103(43): 15975-15980.

Torti, M., E. T. Festetics, et al. (1999). "Clustering of integrin alphallb-beta3 differently regulates tyrosine phosphorylation of pp72syk, PLCgamma2 and pp125FAK in concanavalin Astimulated platelets." Thromb Haemost 81(1): 124-130.

Wagner, D. D. (2005). "New links between inflammation and thrombosis." Arterioscler Thromb Vasc Biol. 25(7): 1321-1324. Epub 2005 Apr 1314.

Yang, R. Y., G. A. Rabinovich, et al. (2008). "Galectins: structure, function and therapeutic potential." Expert Rev Mol Med 10: e17.

Zappelli, C., C. van der Zwaan, et al. (2012). "Novel role for galectin-8 protein as mediator of coagulation factor $V$ endocytosis by megakaryocytes." J Biol Chem 287(11): 8327-8335. 